Keywords: Clinical Index of Stable Febrile Neutropenia; CISNE; nomogram; seemingly stable patients; risk prediction; complications

\title{
A nomogram for predicting complications in patients with solid tumours and seemingly stable febrile neutropenia
}

Paula Jiménez Fonseca ${ }^{1}$, Alberto Carmona-Bayonas ${ }^{\star}, 2$, Ignacio Matos García ${ }^{3}$, Rosana Marcos ${ }^{3}$, Eduardo Castañón ${ }^{4}$, Maite Antonio ${ }^{5}$, Carme Font ${ }^{6}$, Mercè Biosca ${ }^{7}$, Ana Blasco ${ }^{8}$, Rebeca Lozano ${ }^{3}$, Avinash Ramchandani ${ }^{9}$, Carmen Beato ${ }^{10}$, Eva Martínez de Castro ${ }^{11}$, Javier Espinosa ${ }^{12}$, Jerónimo Martínez-García ${ }^{13}$, Ismael Ghanem ${ }^{14}$, Jorge Hernando Cubero ${ }^{15}$, Isabel Aragón Manrique ${ }^{16}$, Francisco García Navalón ${ }^{8}$, Elena Sevillano ${ }^{17}$, Aránzazu Manzano ${ }^{18}$, Juan Virizuela ${ }^{19}$, Marcelo Garrido ${ }^{20}$, Rebeca Mondéjar ${ }^{21}$, María Ángeles Arcusa ${ }^{22}$, Yaiza Bonilla ${ }^{23}$, Quionia Pérez ${ }^{24}$, Elena Gallardo ${ }^{25}$, Maria del Carmen Soriano ${ }^{26}$, Mercè Cardona ${ }^{27}$, Fernando Sánchez Lasheras ${ }^{28}$, Juan Jesús Cruz ${ }^{3}$ and Francisco Ayala ${ }^{2}$ on behalf of the Supportive Care Working Group of the Spanish Society of Medical Oncology (SEOM) and the FINITE investigators

Background: We sought to develop and externally validate a nomogram and web-based calculator to individually predict the development of serious complications in seemingly stable adult patients with solid tumours and episodes of febrile neutropenia (FN).

Patients and methods: The data from the FINITE study $(n=1133)$ and University of Salamanca Hospital (USH) FN registry ( $n=296)$ were used to develop and validate this tool. The main eligibility criterion was the presence of apparent clinical stability, defined as events without acute organ dysfunction, abnormal vital signs, or major infections. Discriminatory ability was measured as the concordance index and stratification into risk groups.

Results: The rate of infection-related complications in the FINITE and USH series was $13.4 \%$ and $18.6 \%$, respectively. The nomogram used the following covariates: Eastern Cooperative Group (ECOG) Performance Status $\geqslant 2$, chronic obstructive pulmonary disease, chronic cardiovascular disease, mucositis of grade $\geqslant 2$ (National Cancer Institute Common Toxicity Criteria), monocytes $<200 / \mathrm{mm}^{3}$, and stress-induced hyperglycaemia. The nomogram predictions appeared to be well calibrated in both data sets (Hosmer-Lemeshow test, $P>0.1$ ). The concordance index was 0.855 and 0.831 in each series. Risk group stratification revealed a significant distinction in the proportion of complications. With a $\geqslant 116$-point cutoff, the nomogram yielded the following prognostic indices in the USH registry validation series: $66 \%$ sensitivity, $83 \%$ specificity, 3.88 positive likelihood ratio, $48 \%$ positive predictive value, and $91 \%$ negative predictive value.

Conclusions: We have developed and externally validated a nomogram and web calculator to predict serious complications that can potentially impact decision-making in patients with seemingly stable FN.

Over the years, in-patient intravenous antibiotic treatment has been demonstrated to be the most effective strategy for patients with postchemotherapy febrile neutropenia (FN). More recently, patients with solid tumours have been seen to present mild episodes associated with clinical stability, particularly when they receive care in the early stages of infection (Carmona-Bayonas

*Correspondence: Dr A Carmona-Bayonas; E-mail: alberto.carmonabayonas@gmail.com 
et al, 2011); hence, not all of them require prolonged hospitalisation for intravenous antibiotics. Consequently, various groups have sought to stratify patients with FN into high- and low-risk groups, in an attempt to individually predict their clinical course and optimise treatment (Talcott et al, 1992; Klastersky et al, 2000; Ahn et al, 2015; Carmona-Bayonas et al, 2015).

The Continuous Care Working Group of the Spanish Society of Medical Oncology (SEOM, for its acronym in Spanish) has recently devised a prognostic classification, called the Clinical Index of Stable Febrile Neutropenia or CISNE, for patients with solid tumours and seemingly stable episodes. CISNE is capable of classifying stable patients according to their risk of serious complications and death more accurately than previous scales (Carmona-Bayonas et al, 2015).

CISNE is used in the same way as the eligibility criteria of most randomised clinical trials that compared in-patient treatment to ambulatory treatment or oral $v s$ intravenous antibiotics and that excluded patients using pragmatic clinical criteria, such as shock, comorbidity, acute organ failure, pneumonia, or oral intolerance (Teuffel et al, 2011; Vidal et al, 2013). These potentially serious situations comprise a starting point for prognostic evaluation, as recommended by the American Society of Clinical Oncology's (ASCO) clinical practice guideline (Flowers et al, 2013).

The CISNE model was designed as an adjuvant tool in decisionmaking, allowing to individually predict the development of serious complications despite the absence of evident risk criteria such as those previously mentioned (Carmona-Bayonas et al, 2015). While CISNE's prognostic potential has been demonstrated, further applicability and efficacy evaluations are needed to generalise its use in the community.

The aim of this study was to develop a nomogram and online calculator capable of generating individualised predictions, and to externally validate this new decision-making support tool to predict serious complications in an independent series of the University of Salamanca Hospital (USH) FN registry.

\section{MATERIALS AND METHODS}

Patients. The nomogram was developed from a prospective cohort of 1133 patients with seemingly stable FN from the FINITE (Evaluación de Factores Pronósticos en Fiebre Neutropénica, Tumour Sólido y Episodios Estables) study. A description of the FINITE study design, data collection, data quality, and eligibility criteria have been reported previously (Carmona-Bayonas et al, 2015). Briefly put, FINITE was a multicentre study conducted in 22 Spanish centres and one Chilean centre between 2012 and 2014. The key eligibility criteria required that the patient be adult, have a solid tumour treated with mild-moderate intensity chemotherapy, not be hospitalised for another reason, not have acute organ failure (renal, cardiac, respiratory) or decompensation of chronic insufficiency, lack septic shock or hypotension (systolic pressure $<90 \mathrm{~mm} \mathrm{Hg}$ ), have no severe infections, and lack other serious complications within the first $3 \mathrm{~h}$ of being diagnosed with FN (see definitions in Table 1).

Validation was carried out with the USH FN registry. The University of Salamanca Hospital is a 900-plus bed tertiary institution, and its Medical Oncology Department is the reference department for an estimated population of 350000 inhabitants. In 2010, this department launched a project to create a database of FN patients. The objective of this registry was to understand the epidemiological characteristics and outcomes after the centre had instituted an ambulatory programme to manage these patients. This registry has been reported previously by the authors (Matos et al, 2014). The hospital's Ethics Review Board approved the registry and written informed consent was obtained from the patients.

The criteria for entry into the registry included patients $(\geqslant 18$ years) with solid tumours or lymphomas treated with chemotherapy who sought emergency medical care for fever $\geqslant 38^{\circ} \mathrm{C}$ and neutrophils $\leqslant 500 / \mathrm{mm}^{3}$ (or $\leqslant 1000 / \mathrm{mm}^{3}$ with an expected decrease $\leqslant 500 / \mathrm{mm}^{3}$ ), regardless of their clinical status. This registry took into account only the first episode suffered by a patient during the study period.

Study design. The FINITE study design has been described previously (Carmona-Bayonas et al, 2015). Insofar as the USH registry is concerned, clinical data sources consisted of information gleaned from both patient and Emergency Department records. This registry was updated periodically by the on-duty oncologists and the people charged with treating these patients. To standardise collection, the USH investigators designed a form containing the following variables: description of patients' clinical status, including vital signs, other medical comorbidities, tumour type and stage, oncological treatment, FN management, microbiology, duration, and outcome. Missing values for any CISNE explanatory model covariates were disallowed, although all predictors were readily available and there were no exclusions for this reason.

Patients in the USH registry were classified as seemingly stable or unstable, as per the same criterion followed in the CISNE-derived series (see Table 2). All individuals remained in observation for at least $6 \mathrm{~h}$. As per the treating physician's judgement, clinically stable patients were given the possibility of receiving direct home care (discharge between 6 and $24 \mathrm{~h}$ ), early discharge $(24-72 \mathrm{~h}$ ), or standard admission (stay $\geqslant 72 \mathrm{~h}$ ).

The primary end point for this analysis was the occurrence of major FN-associated complications in patients believed to be clinically stable. Major complications were defined as hypotension, acute renal, respiratory, or cardiac failure, arrhythmia, major bleeding, delirium, acute abdomen, disseminated intravascular coagulation, and other events (Table 1).

To minimise subjectivity in data interpretation, two specialists in patient support treatment from USH evaluated the presence of complications and CISNE predictors separately, according to detailed instructions and objective definitions of the variables (Table 2). The investigators were not informed as to the CISNE categories, despite the fact that data collection was not blinded.

Observation was a minimum of 7 days from the time of arrival at the USH Emergency Department until the episode had fully resolved. Resolution, in turn, was defined as the disappearance of all signs of infection, recovery from neutropenia $(\geqslant 1500$ neutrophils per $\mathrm{mm}^{3}$ ), and being afebrile for $48 \mathrm{~h}$. In the event that the patient was discharged early or received ambulatory treatment, a home surveillance component was used to cover this period.

Development of the nomogram and web-based calculator. A nomogram is a graphic representation of the solution of an equation that provides a reasonable approximation of the probability of a particular outcome. The CISNE model explanatory covariables consisted of: Eastern Cooperative Group (ECOG) Performance Status $\geqslant 2$, chronic obstructive pulmonary disease, cardiovascular disease, mucositis NCI grade $\geqslant 2$, monocytes $<200 / \mathrm{mm}^{3}$, and stress-induced hyperglycaemia (CarmonaBayonas et al, 2011). To build the nomogram, the models' coefficients were updated and retrained, bearing in mind the entire FINITE series $(n=1133)$. The objective definition of clinical stability, issues regarding inferential generalisability, heterogeneity concerning prevalence of the explanatory covariables, and the model's scope of application have been described previously (Carmona-Bayonas et al, 2015). We used $k$-fold crossvalidation with $k=5$ to limit the risk of overfitting when obtaining the scores associated with each of the nomogram's covariates. We therefore 
Table 1. Key definitions of the study

\begin{tabular}{|c|c|}
\hline Variable & Definition \\
\hline Clinical stability & $\begin{array}{l}\text { Within the first } 3 \mathrm{~h} \text { from the first assessment: (1) no acute organ failure (renal, cardiac, respiratory) or decompensation of chronic } \\
\text { insufficiency, (2) no septic shock or hypotension (systolic pressure }<90 \mathrm{~mm} \mathrm{Hg} \text { ), (3) no known severe infections (see below) and } \\
\text { (4) no other serious complications, included as admission criterion on their own (pulmonary thromboembolism, arrhythmias, } \\
\text { disseminated intravascular coagulation, and bleeding) }\end{array}$ \\
\hline Major infections & $\begin{array}{l}\text { Pneumonia, empyema, peritonitis, cellulitis }>5 \mathrm{~cm} \text {, suspected typhlitis, enteritis } \mathrm{NCl} \text { grade } 3-4 \text {, appendicitis, cholecystitis or } \\
\text { other complicated abdominal infections, meningitis, encephalitis, endocarditis, and pyelonephritis }\end{array}$ \\
\hline Hypotension & Systolic blood pressure remaining at $<90 \mathrm{~mm} \mathrm{Hg}$ and calling for inotropes or aggressive fluid resuscitation \\
\hline Acute respiratory failure & $\begin{array}{l}\text { (1) } \mathrm{O}_{2} \text { saturation }<90 \% \text {, (2) partial pressure } \mathrm{O}_{2} \text { in arterial blood }<60 \mathrm{~mm} \mathrm{Hg} \text {, or (3) partial pressure of } \mathrm{CO}_{2} \text { in arterial blood } \\
\geqslant 45 \mathrm{~mm} \mathrm{Hg}\end{array}$ \\
\hline Acute renal failure & $\begin{array}{l}\text { (1) Increased creatinine by } \geqslant 0.3 \mathrm{mg} \mathrm{dl} \text { within } 48 \mathrm{~h},(2) \text { increased creatinine to } \geqslant 1.5 \text { times baseline in the previous } 7 \text { days, } \\
\text { or (3) urine volume }<0.5 \mathrm{ml} \mathrm{kg}^{-1} \text { per } \mathrm{h} \text { for } 6 \mathrm{~h}\end{array}$ \\
\hline Acute heart failure & Abrupt onset of dyspnoea, pulmonary oedema, and $\mathrm{O}_{2}$ desaturation demanding emergency treatment \\
\hline Arrhythmias & Arrhythmias were deemed complications whenever they altered cardiovascular stability \\
\hline Delirium & Acute, fluctuating disturbance of mental status accompanied by cognitive impairment \\
\hline Major bleeding & $\begin{array}{l}\text { (1) Associated with death, (2) in critical site (intracranial, intraspinal, intraocular, retroperitoneal, or pericardial); (3) associated } \\
\text { with decreased haemoglobin values by } \geqslant 2 \mathrm{~g} \mathrm{dl}^{-1} \text {, or (4) bleeding requiring transfusion of two units of concentrated RBC }\end{array}$ \\
\hline Acute abdomen & Defined as a syndrome due to a variety of disease conditions requiring urgent medical or surgical management \\
\hline COPD & Emphysema, chronic bronchitis, decreased forced expiratory volumes, or need for $\mathrm{O}_{2}$ therapy, corticosteroids, or bronchodilators \\
\hline Chronic cardiovascular disease & $\begin{array}{l}\text { Chronic conditions such as cor pulmonale, heart failure, cardiomyopathy, hypertensive heart disease, arrhythmias, valvular } \\
\text { heart disease, or other structural malformations, at risk of acute exacerbations in the context of FN. Single isolated episodes of } \\
\text { atrial fibrillation in the past are not included here as chronic cardiovascular diseases }\end{array}$ \\
\hline$(\mathrm{SIH})$ & Glucose $\geqslant 121 \mathrm{mg} \mathrm{dl}^{-1}$ or $\geqslant 250 \mathrm{mg} \mathrm{m}^{-2}$ in a diabetic patient (or in patient on steroid treatment) \\
\hline Mucositis $\mathrm{NCl}$ grade $\geqslant 2$ & As a minimum, patchy ulcerations or pseudomembranes, or moderate pain with indication for modified diet \\
\hline
\end{tabular}

conducted five logistic regression models adjusted using a maximum-likelihood estimation to predict the binary outcome (presence or absence of serious complications) on the training subsets over five different random partitions of the data into fivefolds. These models' coefficients served to construct five nomograms. The goodness of fit for each nomogram was verified by means of the Hosmer-Lemeshow test on each subset. Finally, we averaged estimates of all nomogram parameters from the five models to correct and assess the bias thereby generated. The final nomogram (Figure 2) was used to assign a probability of complication to each patient. The same was carried out to develop an online calculator: http://www.iricom.es/prognostictools/Cisne/ inicio.aspx.

To determine the discrimination ability of the final model, we developed 1000 bootstrap replications that were used as internal validation subsets, calculating the model's bias-corrected concordance (c)-index to predict serious complications. To estimate the optimal cutoff point of the model, predictions were dichotomised by Youden's J index (Youden, 1950).

External validation of the nomogram in the USH registry. All patients from USH who met the criterion of clinical stability were assessed using this nomogram (Ghanem et al, 2014). The calibration and discriminatory ability were externally validated in clinically stable patients from the USH registry. First, we used a bootstrapping procedure with 2000 replications to get overfittingcorrected estimates of predicted $v s$ observed values to evaluate the nomogram's calibrations. The absolute mean and squared errors and the 0.9 quantile of the absolute error were calculated. Then, we computed a summary of the c-index of this validation subset as a measure of discrimination. Based on previous research, around 300 patients would be sufficient for this analysis (Peduzzi et al, 1996). All statistical analyses were performed using R software, including the rms package version 4.2-1 (R Development Core Team, 2014; Harrell et al, 2015).

\section{RESULTS}

Patients. Between the two registries, a total of 1952 patients with FN were screened, of which $73 \%$ of the FINITE cohort $(n=1133)$ and $76 \%$ of the USH registry $(n=296)$ were eligible for analysis. Figure 1 is a flowchart of both databases. Table 2 presents the descriptive statistics of each series. There are no differences between both series with respect to age (median of 60 years in both), functional status, and tumour stage. The distribution of tumours and episodes is quite similar. The most remarkable differences between the series lie in the fact that there was greater prophylaxis with granulocyte-colony-stimulating factor (G-CSF) in the FINITE cohort $(27 \%$ vs $13 \% ; P<0.001)$ and prior antibiotic use (18\% vs 5\%; $P<0.001)$. In contrast, the USH patients present a higher rate of direct ambulatory care $(30 \% v s 11 \%, P<0.0002)$ or treatment with oral antibiotics $(36 \%$ vs $10 \%, P<0.0002)$.

Outcomes. The multicentre FINITE series revealed marginally lower rates of serious complications and mortality: 13.4\% (95\% CI, $11.5-15.5)$ and $1.8 \%(95 \% \mathrm{CI}, 1.1-2.7)$, respectively. In comparison, the rate of infection-related serious complications and death in seemingly stable patients in the USH registry was $18.6 \%(95 \%$ CI, 14.6-23.4) and $2.7 \%$ (95\% CI, 1.3\%-5.2\%), respectively. In this registry, the most common complications were shock $(n=22)$, acute respiratory failure $(n=11)$, major bleeding $(n=5)$, and cardiac insufficiency $(n=3)$. In the $108 \mathrm{USH}$ patients who were discharged early or directly, $5 \%(n=6)$ were readmitted because of complications. These complications were hypotension in two cases, and respiratory failure, mucositis, anaemia, and intestinal occlusion in one case each. None of the patients who were readmitted died. In contrast, the death rate was significantly higher among the ineligible USH patients (unstable): 18.1\% (95\% CI, 11.6-27).

Development of a nomogram based on the FINITE data set. First, we developed the prognostic nomogram for estimating 
Table 2. Comparison of baseline characteristics in the study cohorts

\begin{tabular}{|c|c|c|c|}
\hline Characteristics & FINITE series, $n=1133(\%)$ & USH registry, $n=296(\%)$ & $P$-value \\
\hline Age (years), $\geqslant 65$ & $411(36 \%)$ & $105(35 \%)$ & NS \\
\hline Male gender & $520(45 \%)$ & $127(43 \%)$ & NS \\
\hline COPD & $151(13 \%)$ & 27 (9\%) & NS \\
\hline Chronic cardiovascular disease & $86(8 \%)$ & $28(9 \%)$ & NS \\
\hline ECOG PS $\geqslant 2$ & $198(17 \%)$ & $44(15 \%)$ & NS \\
\hline Mucositis $\mathrm{NCl}$ grade $\geqslant 2$ & 207 (18\%) & $29(10 \%)$ & 0.0005 \\
\hline Monocytes $<200 / \mathrm{mm}^{3}$ & 537 (47\%) & $126(43 \%)$ & NS \\
\hline Stress-induced hyperglycaemia & 351 (31\%) & 87 (29\%) & NS \\
\hline Prophylactic G-CSF & 302 (27\%) & $38(13 \%)$ & $<0.0002$ \\
\hline Previous antibiotics, $<1$ month & $202(18 \%)$ & $16(5 \%)$ & $<0.0002$ \\
\hline \multicolumn{4}{|l|}{ Malignant disease } \\
\hline Breast cancer & $391(35 \%)$ & 114 (39\%) & NS \\
\hline Lung cancer & $234(21 \%)$ & $61(21 \%)$ & NS \\
\hline Oesophagus-gastric cancer & 80 (7\%) & $16(5 \%)$ & NS \\
\hline Colorectal cancer & $59(5 \%)$ & $15(5 \%)$ & NS \\
\hline Sarcoma & $50(4 \%)$ & $17(6 \%)$ & NS \\
\hline Germ-cell cancer & $26(2 \%)$ & 9 (3\%) & NS \\
\hline Other & $293(26 \%)$ & $64(22 \%)$ & 0.0003 \\
\hline Tumour stage IV & 517 (46\%) & 140 (47\%) & NS \\
\hline \multicolumn{4}{|l|}{ Foci of infection } \\
\hline Enteritis & $143(13 \%)$ & 27 (9\%) & NS \\
\hline Respiratory tract & 293 (26\%) & $76(26 \%)$ & NS \\
\hline Urinary tract & 67 (6\%) & 32 (11\%) & 0.003 \\
\hline Mucositis & 132 (12\%) & 27 (9\%) & NS \\
\hline No infectious focus & 384 (34\%) & $124(42 \%)$ & 0.01 \\
\hline Other & $114(10 \%)$ & $10(3 \%)$ & 0.0003 \\
\hline \multicolumn{4}{|l|}{ Patient management } \\
\hline Outpatient & $122(11 \%)$ & 90 (30\%) & $<0.0002$ \\
\hline Early discharge & 215 (19\%) & $21(7 \%)$ & $<0.0002$ \\
\hline In-patient & 796 (70\%) & $185(63 \%)$ & 0.01 \\
\hline Initial route of antibiotics, oral & $111(10 \%)$ & $106(36 \%)$ & $<0.0002$ \\
\hline Neutrophils $<100 / \mathrm{mm}^{3}$ & $500(44 \%)$ & $68(23 \%)$ & $<0.0002$ \\
\hline
\end{tabular}

the risk of serious complications. Figure 2 illustrates this nomogram. Actual proportions deviated by $<1 \%$ from the predictions (Figure 3). The Hosmer-Lemeshow test showed good fit of the model for the entire series, and separately for each of the five subsets used to create it. Youden's $J$ index corresponds to $\geqslant 116$ points (equivalent to a risk of serious complications $\geqslant 13 \%$ ), which is considered the optimal cutoff value for high risk. The distribution of prognostic categories by nomogram scores is presented in Table 3. Across the 1000 bootstrap replications, the bootstrap optimism-corrected $c$-index for predicting serious complications was 0.855 (95\% CI, 0.823-0.887).

Validation of the nomogram in the USH registry. We then applied the nomogram to the subgroup of 296 apparently stable USH patients. Table 3 shows how the complications and mortality rates go up as the nomogram score increases $(P<0.001)$. With a cutoff of $\geqslant 116$, the nomogram has $66 \%$ sensitivity, $83 \%$ specificity, 3.88 positive likelihood ratio, 0.41 negative likelihood ratio, $48 \%$ positive predictive value, and $91 \%$ negative predictive value. A c-index of 0.831 (95\% CI, 0.782-0.871) was displayed, denoting good discriminatory ability. The nomogram's predictions appear to be well calibrated compared with those observed in the USH registry with a mean absolute error of $3.6 \%$ (Figure 4 ). The Hosmer-Lemeshow goodness-of-fit test $\left(\chi^{2} 6.01 ; P=0.3\right)$ also exhibited appropriate calibration (Figure 4). Notably, in the subgroup that was readmitted after direct or early discharge $(n=6), 5(83 \%)$ had a score $\geqslant 116$ on the CISNE nomogram (high risk).

\section{DISCUSSION}

This study describes the development of a nomogram to predict prognosis in patients with seemingly stable FN. The criterion for application of the CISNE nomogram is initial apparent clinical stability as defined by the absence of serious complications, extensive infection, shock, and acute organ failure at the time of first evaluation. The nomogram assigns a predicted probability of serious complications based on the CISNE model's explanatory covariables (ECOG PS $\geqslant 2$, chronic obstructive pulmonary disease, cardiovascular disease, mucositis NCI grade $\geqslant 2$, monocytes $<200 / \mathrm{mm}^{3}$, and stress-induced hyperglycaemia). This nomogram has been applied to an external validation series from a registry of consecutive cases of FN at the USH. In this series, it has proven reasonable calibration and good discriminatory ability to predict serious complications.

Although the CISNE nomogram was designed chiefly to predict the likelihood of serious complications in stable patients initiating in-patient therapy, the USH data confirm that a two-stage classification similar to that used in the FINITE study (exclusion criteria plus the CISNE nomogram) comprise a safe method by which to select low-risk patients when the screening takes place within 6 to $24 \mathrm{~h}$ from the time of arrival at the Emergency Department.

The USH series reveals that the absence of baseline criteria of severity (apparent clinical stability) is efficacious when selecting patients with relatively low risk of complications and death; for 


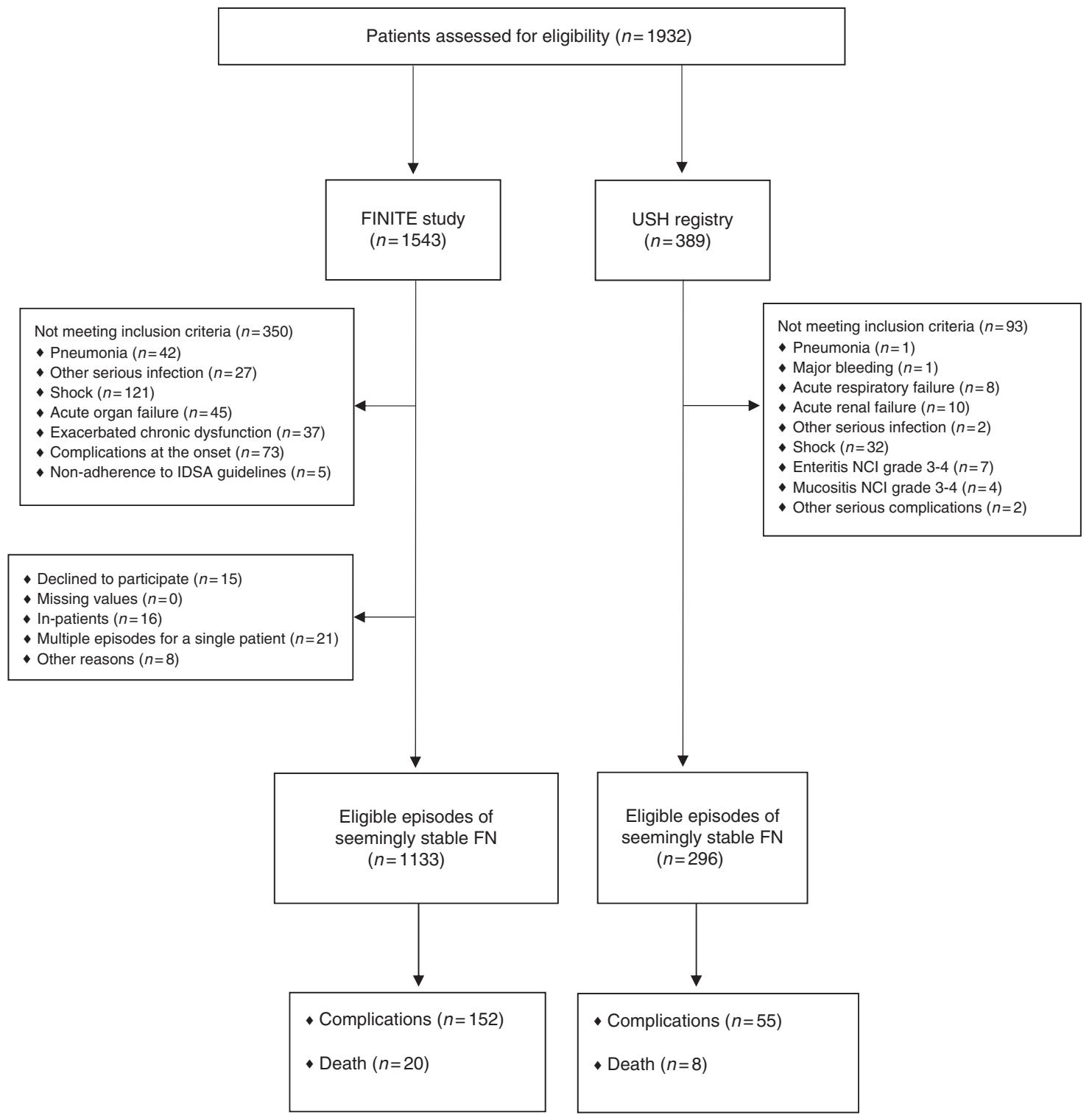

Figure 1. Flow diagram of the FINITE and USH series.

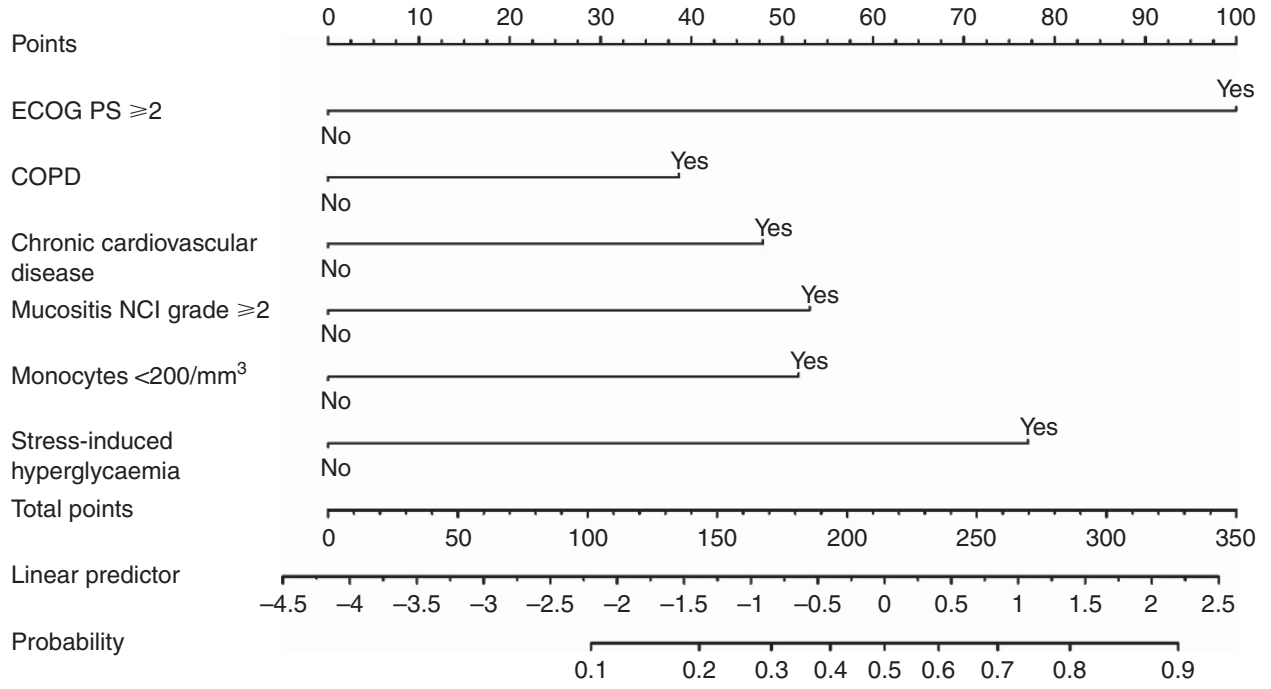

Figure 2. The CISNE nomogram. COPD = chronic obstructive pulmonary disease; ECOG PS = Eastern Cooperative Oncology Group Performance Status; $\mathrm{NCl}=$ National Cancer Institute. 


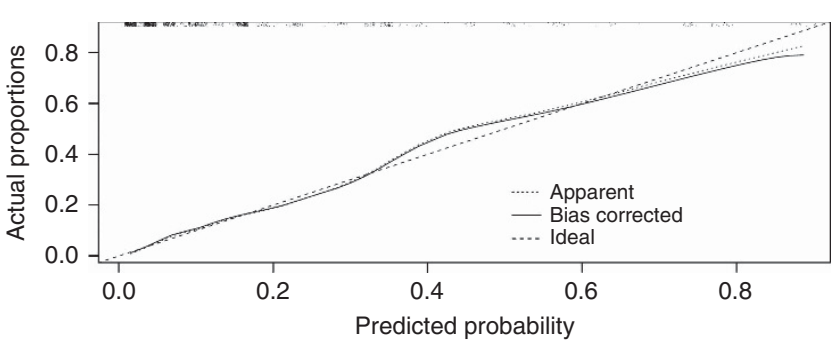

Figure 3. Calibration plot showing predicted probability vs actual (observed) frequencies of serious complications in clinically stable patients from the FINITE study $(n=1133)$. (Bootstrap procedure with 2000 repetitions; mean absolute error $=0.008$; mean squared error $=0.00021 ; 0.9$ quantile of absolute error $=0.014$. Distribution of predicted risks of patients is shown at top of the figure.)

Table 3. Distribution of risk classes and outcomes derived from the FINITE and USH series ${ }^{a}$

\begin{tabular}{|c|c|c|}
\hline \multicolumn{3}{|c|}{$\%(95 \%$ confidence interval) } \\
\hline & $\begin{array}{l}\text { FINITE series, } \\
n=1133(\%)\end{array}$ & $\begin{array}{l}\text { USH registry, } \\
n=296(\%)\end{array}$ \\
\hline \multicolumn{3}{|c|}{ Distribution of nomogram points } \\
\hline $0-57$ & $49.6(46.7-52.6)$ & $55.1(49.3-60.6)$ \\
\hline 58-115 & $19.2(17.0-21.6)$ & $18.5(14.5-23.4)$ \\
\hline $116-175$ & $17.3(15.2-19.6)$ & $15.5(11.8-20.1)$ \\
\hline $176-231$ & $9.7(8.1-11.5)$ & $8.1(5.5-11.7)$ \\
\hline$\geqslant 232$ & $4.1(3.1-5.3)$ & $2.7(1.3-5.2)$ \\
\hline \multicolumn{3}{|c|}{ Complications by nomogram points } \\
\hline $0-57$ & $2.3(1.3-3.9)$ & $4.2(2.0-8.5)$ \\
\hline 58-115 & $9.6(6.3-14.2)$ & $20(11.5-32.3)$ \\
\hline $116-175$ & $17(13.1-23.8)$ & $37.8(26.3-53.5)$ \\
\hline $176-231$ & $47.2(38.1-56.5)$ & $50(31.4-68.5)$ \\
\hline$\geqslant 232$ & $67.3(52.9-79.1)$ & $100(67-100)$ \\
\hline \multicolumn{3}{|c|}{ Mortality by nomogram points } \\
\hline $0-57$ & $0.1(0.03-1.0)$ & $0.6(0.1-3.3)$ \\
\hline $58-115$ & $2.2(0.9-5.2)$ & $1.8(0.3-9.6)$ \\
\hline $116-175$ & $2.5(1.1-5.8)$ & $2.2(3.9-11.5)$ \\
\hline $176-231$ & $4.5(1.9-10.2)$ & $8.3(2.3-25.8)$ \\
\hline$\geqslant 232$ & $8.7(3.4-20.3)$ & $37.5(13.6-69.4)$ \\
\hline \multicolumn{3}{|c|}{$\begin{array}{l}\text { Abbreviations: FINITE = Evaluación de Factores Pronósticos en Fiebre Neutropénica, } \\
\text { Tumour Sólido y Episodios Estables; USH = University of Salamanca Hospital. } \\
\text { a In the FINITE series, Youden's }(J \text { index was } \geqslant 116 \text { points (high risk). The cutoffs used to } \\
\text { create the five prognostic categories are multiples of half of Youden's index. }\end{array}$} \\
\hline
\end{tabular}

instance, infection-related mortality was significantly lower in seemingly stable patients in comparison with clinically unstable subjects ( $3 \%$ vs $18 \%)$. However, the absence of severity criteria at the onset does not fully rule out the possibility that the neutropenic patient will develop serious complications, which occurred in $18 \%$ of the patients deemed stable and even in 5.5\% of those who received direct or early discharged in the USH cohort.

In light of the aforementioned, we believe that a CISNE-based nomogram can contribute to improve exclusion criteria for management in these patients, as well as their classification, or decision-making regarding oral or outpatient treatment.

The nomogram has several limitations that must be indicated to optimise its use. While the model's functioning was robust in a subsequent update and its explanatory covariables therefore appear to be appropriate in different contexts, one must always be mindful of the possibility of uncommon risk factors, for example, cirrhosis, malnutrition, chronic renal disease, or other pathologies or situations. Although patients with lymphomas were included, there were so few cases $(n=21)$ as to advise caution when dealing with this population. The USH registry's sample size depended on patient volume, but the series contains $\sim 91 \%$ of

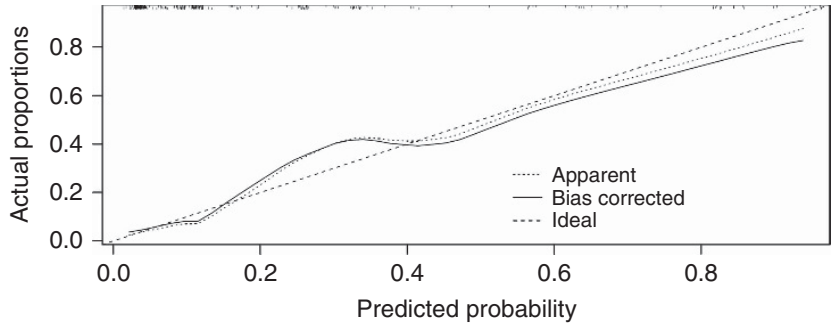

Figure 4. Calibration plot showing predicted probability vs actual (observed) proportions of serious complications in clinically stable patients from the USH registry $(n=296)$. (Bootstrap procedure with 2000 repetitions; mean absolute error $=0.036$; mean squared error $=0.00291 ; 0.9$ quantile of absolute error $=0.111$. Distribution of predicted risks of patients is shown at top of the figure.)

the events generally recommended for studies such as this one (Peduzzi et al, 1996). Similarly, it is possible that the nomogram's discriminatory ability might be enhanced in the future by incorporating new variables. Given their extraordinary variability, it is important that we attempt to integrate patients' specific infection and oncological status. Nevertheless, these six variables appear to be germane and discriminatory in the three series analyzed. The model appears to be well calibrated across the spectrum of severity despite which a slight underestimation of risk was seen in the range between 20 and $40 \%$ of predicted probabilities of the USH cohort. Although the eligibility criteria for oral, outpatient management in the USH registry were similar to those of the Infectious Disease Society of America (IDSA) clinical practice guidelines (Freifeld et al, 2011), the decision as to where to treat fell ultimately to the treating physician, who had to factor in each patient's particular risk factors, or other family, psychological, or social aspects. The USH series also suggests that a percentage of serious complications can be detected only by increasing the screening period beyond $3 \mathrm{~h}$ (e.g. by in-hospital observation for 6-24h).

Finally, how the CISNE model would be used within the current classification systems recommended by clinical guidelines must be elucidated, as well as the differences with respect to other existing models, such as the Talcott and Multinational Association for Supportive Care in Cancer (MASCC) score (Talcott et al, 1992; Klastersky et al, 2000). At present, the MASCC score is the most widely validated and recommended to identify low-risk patients, although never as a stand-alone selection method, but rather in combination with exclusion criteria similar to the ones proposed in this article (Flowers et al, 2013). However, our group has demonstrated that the CISNE score is better able to discriminate than the MASCC model when used in apparently stable patients with solid tumours: areas under the receiver operating characteristic curves 0.721 (95\% CI, 0.669-0.768) for MASCC and 0.868 (95\% CI, 0.827-0.903) for CISNE $(P=0.002)$ (Carmona-Bayonas et al, 2015).

Several methodological issues hinder the applicability of the MASCC model in daily clinical practice. Most notable among them are that the heaviest weighted predictor, hypotension, is also the most common outcome; that a relevant percentage had acute leukaemia or bone marrow transplant, limiting its application to patients with solid tumours; or that many patients were initially haemodynamically unstable and could therefore never be classified as low risk, despite their MASCC score.

The most obvious advantage of the CISNE model over the previously proposed ones is that the two-step triage process improves patient safety, reinforcing decision-making without added complexity. CISNE seeks to decrease the uncertainty linked to the diminished inflammatory response due to immunosuppression, which undermines the clinician's ability to detect 
complications early on. As a final point, CISNE is a safe method because, unlike others, its prime purpose is not to release patients early, but just the opposite - to keep them from occurring until the apparent stability has been confirmed as real after an appropriate time of in-hospital observation. Therefore, the results of our study are mainly applicable to avoid early discharge of patients with cancer beginning in-patient therapy. In light of our data, we suggest that patients experiencing seemingly stable episodes with a CISNE nomogram score $\geqslant 116$ points (equivalent to a risk of serious complications $\geqslant 13 \%$ ) should not be sent home until they have proven to be truly stable and blood cultures have been examined. On the contrary, we emphasise that this nomogram should not be used so much to select low-risk patients for direct outpatient treatment. Nevertheless, well-conducted clinical trials are required to endorse that this prognostic classification can be safely and efficaciously associated with therapeutic classes.

In short, we report a nomogram to predict complications in seemingly stable patients with solid tumour who present $\mathrm{FN}$ and who commence in-patient treatment. This tool has been developed in two different series that, together, comprise more than 1400 eligible patients and subsequently validated using an external database. The nomogram can therefore be useful for individualising patient care in terms of treatment and place, in addition to interpreting other series and future studies in this field.

\section{ACKNOWLEDGEMENTS}

Natalia Cateriano and Miguel Vaquero (IRICOM SL); Priscilla Chase Duran (PCD Translations); Jaime Baladrón (Curso MIR Asturias); Luis Aulló (Design and Illustration); the Supportive Care Working Group of the Spanish Society of Medical Oncology (SEOM) and SEOM staff whose enthusiastic work made this study possible. This is an academic study. No financial support has been received from external sources. The web calculator was funded by the sales of the book 'Comer para vencer el cáncer', written by one of the article's authors (PJF), and by altruistic donations given by the investigators themselves.

\section{CONFLICT OF INTEREST}

The authors declare no conflict of interest.

\section{AUTHOR CONTRIBUTIONS}

Conception and design: Alberto Carmona-Bayonas, Paula JiménezFonseca and Juan Virizuela Echaburu. Financial support: Alberto Carmona-Bayonas, Paula Jiménez-Fonseca and Juan Virizuela Echaburu. Administrative support: Alberto Carmona-Bayonas, Paula Jiménez-Fonseca, Juan Virizuela Echaburu and Juan Jesús Cruz. Provision of study materials or patients: Alberto CarmonaBayonas, Paula Jiménez-Fonseca, Maite Antonio, Carme Font, Mercè Biosca, Avinash Ramchandani, Jerónimo Martínez-García, Jorge Hernando Cubero, Javier Espinosa, Ismael Ghanem, Carmen Beato, Ana Blasco, Marcelo Garrido, Rebeca Mondéjar, María Ángeles Arcusa Lanza, Isabel Aragón Manrique, Aránzazu Manzano, Elena Sevillano, Eduardo Castañón, Mercé Cardona, Elena Gallardo Martín, Quionia Pérez Armillas, Yaiza Bonilla, Ignacio Matos García, Rosana Marcos Sánchez, Rebeca Lozano Mejorada and Francisco García Navalón. Collection and assembly of data: Alberto Carmona-Bayonas and Paula Jiménez-Fonseca. Data analysis and interpretation: Alberto Carmona-Bayonas, Paula Jiménez-Fonseca, Fernando Sánchez Lasheras and Francisco Ayala de la Peña. Manuscript writing: Alberto Carmona-Bayonas, Paula
Jiménez-Fonseca, Fernando Sánchez Lasheras and Francisco Ayala de la Peña. Final approval of manuscript: All authors.

\section{REFERENCES}

Ahn S, Lee Y-S, Lee J-L, Lim KS, Yoon S-C (2015) A new prognostic model for chemotherapy-induced febrile neutropenia. Int J Clin Oncol 1-7.

Carmona-Bayonas A, Gómez J, González-Billalabeitia E, Canteras M, Navarrete A, Gonzálvez ML, Vicente V, Ayala de la Peña F (2011) Prognostic evaluation of febrile neutropenia in apparently stable adult cancer patients. Br J Cancer 105: 612-617.

Carmona-Bayonas A, Jiménez-Fonseca P, Virizuela Echaburu J, Antonio M, Font C, Biosca M, Ramchandani A, Martínez J, Hernando Cubero J, Espinosa J, Martínez de Castro E, Ghanem I, Beato C, Blasco A, Garrido M, Bonilla Y, Mondéjar R, Arcusa Lanza MÁ, Aragón Manrique I, Manzano A, Sevillano E, Castañón E, Cardona M, Gallardo Martín E, Pérez Armillas Q, Sánchez Lasheras F, Ayala de la Peña F (2015) Prediction of serious complications in patients with seemingly stable febrile neutropenia: validation of the Clinical Index of Stable Febrile Neutropenia in a prospective cohort of patients from the FINITE study. J Clin Oncol 33: 465-471.

Flowers CR, Seidenfeld J, Bow EJ, Karten C, Gleason C, Hawley DK, Kuderer NM, Langston AA, Marr KA, Rolston KVI, Ramsey SD (2013) Antimicrobial prophylaxis and outpatient management of fever and neutropenia in adults treated for malignancy: American Society of Clinical Oncology Clinical Practice Guideline. J Clin Oncol 31: 794-810.

Freifeld AG, Bow EJ, Sepkowitz KA, Boeckh MJ, Ito JI, Mullen CA, Raad II, Rolston KV, Young J-AAH, Wingard JR. Infectious Diseases Society of America (2011) Clinical practice guideline for the use of antimicrobial agents in neutropenic patients with cancer: 2010 update by the infectious diseases society of america. Clin Infect Dis 52: e56-e93.

Ghanem I, Antonio M, Garrido M, Martínez-García J, Font C, Ramchandani, Avinash, Biosca M, Beato C, Martínez de Castro E, Castañón E, Virizuela Echaburu J, Espinosa J, Sevillano E, Aragón Manrique I, Carmona-Bayonas A (2014) A nomogram for predicting serious complications in patients with solid tumors and apparently stable febrile neutropenia: prospective data on 781 consecutive episodes from the finite study. J Clin Oncol 32(Suppl 31): abstr 165.

Harrell Jr FE, Maintaner Harrell Jr FE (2015) Package ' $r m s$ '. Vanderbilt University: Nashville, TN, USA.

Klastersky J, Paesmans M, Rubenstein EB, Boyer M, Elting L, Feld R, Gallagher J, Herrstedt J, Rapoport B, Rolston K, Talcolt J (2000) The multinational association for supportive care in cancer risk index: a multinational scoring system for identifying low-risk febrile neutropenic cancer patients. J Clin Oncol 18: 3038-3051.

Matos I, Lozano Mejorada R, Rodríguez C, Alfonso S, Marcos R, Vidal R, Guillén C, Noguerido A, Rúa O, Del Barco E, Navarro L, Cruz J (2014) Analysis of clinical factors for ambulatory management in patients with febrile neutropenia. ESMO Meeting, p 1506.

Peduzzi P, Concato J, Kemper E, Holford TR, Feinstem AR (1996) A simulation study of the number of events per variable in logistic regression analysis. J Clin Epidemiol 49: 1373-1379.

R Development Core Team (2014) R: A Language and Environment for Statistical Computing. R Foundation for Statistical Computing: Vienna, Austria, 2012.

Talcott JA, Siegel RD, Finberg R, Goldman L (1992) Risk assessment in cancer patients with fever and neutropenia: a prospective, two-center validation of a prediction rule. J Clin Oncol 10: 316-322.

Teuffel O, Ethier MC, Alibhai SMH, Beyene J, Sung L (2011) Outpatient management of cancer patients with febrile neutropenia: a systematic review and meta-analysis. Ann Oncol 22: 2358-2365.

Vidal L, Ben Dor I, Paul M, Eliakim-Raz N, Pokroy E, Soares-Weiser K, Leibovici L (2013) Oral versus intravenous antibiotic treatment for febrile neutropenia in cancer patients. Cochrane Database Syst Rev 10: CD003992. Youden WJ (1950) Index for rating diagnostic tests. Cancer 3: 32-35.

This work is published under the standard license to publish agreement. After 12 months the work will become freely available and the license terms will switch to a Creative Commons AttributionNonCommercial-Share Alike 4.0 Unported License. 
${ }^{1}$ Medical Oncology Department, Hospital Universitario Central de Asturias, Oviedo, Spain; ${ }^{2}$ Hematology and Medical Oncology Department, Hospital Universitario Morales Meseguer, Calle Marqués de los Vélez s/n, Murcia 30008, Spain; ${ }^{3}$ Medical Oncology Department, Hospital Universitario de Salamanca, Salamanca, Spain; ${ }^{4}$ Medical Oncology Department, Clínica Universidad de Navarra, Pamplona, Spain; ${ }^{5}$ Medical Oncology Department, ICO Duran i Reynals, Barcelona, Spain; ${ }^{6}$ Medical Oncology Department, Hospital Clínic de Barcelona, Barcelona, Spain; ${ }^{7}$ Medical Oncology Department, Hospital Universitari Vall d'Hebron, Barcelona, Spain; ${ }^{8}$ Medical Oncology Department, Hospital General Universitario de Valencia, Valencia, Spain; ${ }^{9}$ Medical Oncology Department, Hospital Universitario Insular de Gran Canaria, Las Palmas, Spain; ${ }^{10}$ Medical Oncology Department, Hospital NISA Aljarafe, Sevilla, Spain; ${ }^{11}$ Medical Oncology Department, Hospital Universitario Marqués de Valdecilla, Santander, Spain; ${ }^{12}$ Medical Oncology Department, Hospital General Universitario de Ciudad Real, Ciudad Real, Spain; ${ }^{13}$ Medical Oncology Department, Hospital Universitario Virgen de la Arrixaca, Murcia, Spain; ${ }^{14}$ Medical Oncology Department, Hospital Universitario La Paz, Madrid, Spain; ${ }^{15}$ Medical Oncology Department, Hospital Universitario Miguel Servet, Zaragoza, Spain; ${ }^{16}$ Medical Oncology Department, Hospital Juan Ramón Jiménez, Huelva, Spain; ${ }^{17}$ Medical Oncology Department, Hospital Universitario Son Espases, Palma de Mallorca, Spain; ${ }^{18}$ Medical Oncology Department, Hospital Universitario Clínico San Carlos, Madrid, Spain; ${ }^{19}$ Medical Oncology Department, Hospital Universitario Virgen de Macarena, Sevilla, Spain; ${ }^{20}$ Medical Oncology Department, Pontificia Universidad Católica de Chile, Santiago de Chile, Chile; ${ }^{21}$ Medical Oncology Department, Hospital Universitario de La Princesa, Madrid, Spain; ${ }^{22}$ Medical Oncology Department, Consorci Sanitari de Terrassa, Barcelona, Spain; ${ }^{23}$ Intensive Care Unit, Hospital General Universitario Santa Lucía, Cartagena, Spain; ${ }^{24}$ Medical Oncology Department, Hospital Clínico Universitario de Valladolid, Valladolid, Spain; ${ }^{25}$ Medical Oncology Department, Complejo Universitario de Pontevedra, Pontevedra, Spain; ${ }^{26}$ Medical Oncology Department, Hospital Virgen de la Luz de Cuenca, Madrid, Spain; ${ }^{27}$ Internal Medicine Department, Hospital de Tortosa Verge de la Cinta, Tarragona, Spain and ${ }^{28}$ Department of Construction and Manufacturing Engineering, University of Oviedo, Spain 